\title{
EDITORIAL
}

\section{FRAILTY AND AGING}

\section{S. SOURDET ${ }^{1}$, M.E. ROUGE-BUGAT ${ }^{3}$, B. VELLAS $^{1,2}$, F. FORETTE $^{4}$}

1. Gerontopole, Toulouse, France; 2. Inserm U 1027, Alzheimer's Disease Research and Clinical Center, Toulouse, University Hospital, France; 3. Department of Family Medicine, University of Toulouse, France; 4. AP-HP, Hôpital Broca, Université René Descartes, Paris, France

Aging is associated with functional decline and loss of autonomy, and age is a major risk factor for a wide spectrum of clinical conditions, including cardiovascular disease, cognitive impairment, and physical disability. As life expectancy continues to rise, the maintain of physical functioning advanced age has emerged as a major clinical and public health priority.

It is estimated that by $2025,1.2$ billion people worldwide will be over 60 years old (1), with similar prevalence in Europe (2). In France, men and women aged 50 years old can expect to live free of disability respectively for another 18.0 years and 19.7 years. These data are similar among all $25 \mathrm{EU}$ countries where healthy aging in men and women is expected until age 67 and 68 years, respectively. However, in adults age 50 years and older, approximately $40 \%$ of men and $46 \%$ of women, will experience some functional limitation (1).

Frailty has been operationally defined by Fried and colleagues based on a phenotypic definition in older adults. From a clinical perspective, frailty is marked by three or more of the following criteria: unintentional weight loss in the past year ( $>4 \mathrm{~kg}$ ), self-reported exhaustion, weakness (measured by loss of grip strength), slow walking speed, low physical activity. The term "frailty" is commonly used to indicate a state of high vulnerability to negative health-related outcomes, such as falls, physical and cognitive decline, hospitalization, physical disability, and mortality. Clinically, frailty is a loss of functional homeostasis resulting in a pathophysiological state characterized by the accumulation of molecular, cellular, and tissue damage in different organ systems. When function has already been lost due to injury or illness, frailty can confound the loss and lead to disability. Frailty and functional decline are frequent consequences of aging, but their etiologies remain unclear. Older adults may experience progressive decline in function and develop disabilities without any clinical explanation $(3,4)$. The frailty syndrome is possibly a result of the early stages and non-specific symptoms of both age-related diseases and complex pathophysiological processes not yet identified. Thus, early interventions aimed at preventing the progression of frailty and disability are critical in older adults.

An intermediate status, "pre-frail," is characterized by the presence of one or two of the criteria defined above. Pre-frail older adults are at an increased risk of adverse outcomes, as well as increased risk of meeting criteria for frailty in three to four years. Together, pre-frail and frail adults represent almost half of the population aged 60 and older. In France and other countries of the EU, previous epidemiological studies have reported rates of frailty and pre-frailty in approximately $15 \%$ and 50\% in community-dwelling adults aged 65 years and older, respectively (5). Although these groups are at an increased risk for disease and disability, they are also the most to benefit from interventions. More specifically, interventions designed for older adults with pre-frailty might reduce the risk of progression to a more serious condition.

Given the global trend of an aging population, increased efforts are necessary to identify ways to better predict risk of frailty and interventions to prevent the occurrence of frailty in the elderly. Frailty is a medical syndrome characterized by multiple changes and deficits in different biological systems, including sarcopenia (i.e., the loss of both muscle mass and strength), osteoporosis (i.e., low bone mineral density), neuroendocrine and metabolic dysregulation, elevation in inflammatory factors, malnutrition, and cardiovascular abnormalities (6). Some of these manifestations have been largely studied in older adult populations. For instance, a slow gait speed is a criterion for the diagnosis of sarcopenia (7). People with slow gait speed have also been found to be more likely to exhibit several other age-related diseases (e.g., Mild Cognitive Impairment and osteoporosis) (8). Thus, gait speed can be used as a reliable measure for frailty (9). Despite the empirical evidence for certain biological etiologies for frailty, there are many factors that warrant further investigation to better understand their role in aging and frailty in older adults. For example, Kaya and Saurat (10) demonstrated that dermatoporosis, a condition of frail aging skin, frequently occurs in older adults, and more specifically in those who are frail. It is possible to restore the function of the skin after dermatoporosis begins; however, if it is left untreated it can produce ulceration and hypercatabolism. It can also contribute to pain, functional impairment, slow gait speed, and further disability.

Frailty and concurrent age-related diseases are key targets for the prevention of disability in older age. Nearly half of adults over 50 are at risk of developing some functional limitations, however, healthy aging is possible well beyond this age and into later life. Although some functional decline is expected during the aging process, older adults are at higher risk of developing diseases and disabilities associated with changes that occur with aging. Established criteria have improved our ability to identify frailty and pre-frailty, yet there are no empirically supported interventions to delay or prevent this syndrome. Continuous efforts and well-designed intervention studies are needed to reduce frailty and consequent disability in our rapidly increasing aging population. 


\section{FRAILTY AND AGING}

\section{References}

1. C. Jagger and al. Lancet Inequalities in healthy life years in the 25 countries of the European Union in 2005: a cross-national meta-regression analysis. Lancet 2008 372: 2124-31

2. Garcia-Garcia FJ, Gutierrez Avila G, Alfaro-Acha A, Amor Andres MS, De Los Angeles De La Torre Lanza M, Escribano Aparicio MV, Humanes Aparicio S, Larrion Zugasti JL, Gomez-Serranillo Reus M, Rodriguez-Artalejo F, RodriguezManas L.The prevalence of frailty syndrome in an older population from Spain. The toledo study for healthy aging. J Nutr Health Aging. 2011;15(10):852-6.

3. Mitnitski A, Fallah N, Rockwood MR, Rockwood K Transitions in cognitive status in relation to frailty in older adults: a comparison of three frailty measures. J Nutr Health Aging. 2011;15(10):863-7.

4. Fried LP, Young Y, Rubin G, Bandeen-Roche K; WHAS II Collaborative Research Group. Self-reported preclinical disability identifies older women with early declines in performance and early disease. J Clin Epidemiol. 2001 Sep;54(9):889-901.

5. Santos-Eggimann B, Cuénoud P, Spagnoli J, Junod J. Prevalence of frailty in middleaged and older community-dwelling Europeans living in 10 countries. J Gerontol A Biol Sci Med Sci. 2009 Jun;64(6):675-81. Epub 2009 Mar 10.
6. Fulop T, Larbi A, Witkowski JM, McElhaney J, Loeb M, Mitnitski A, Pawelec G. Aging, frailty and age-related diseases. Biogerontology. 2010 Oct;11(5):547-63. Epub 2010 Jun 18.

7. Cruz-Jentoft, A. J., J. P. Baeyens, et al. "Sarcopenia: European consensus on definition and diagnosis: Report of the European Working Group on Sarcopenia in Older People." Age Ageing 39(4): 412-23.

8. Abellan van Kan G, Rolland Y, Andrieu S, Bauer J, Beauchet O, Bonnefoy M, Cesari M, Donini LM, Gillette Guyonnet S, Inzitari M, Nourhashemi F, Onder G, Ritz P, Salva A, Visser M, Vellas B. Gait speed at usual pace as a predictor of adverse outcomes in community-dwelling older people an International Academy on Nutrition and Aging (IANA) Task Force. J Nutr Health Aging. 2009 Dec;13(10):881-

9. Abellan van Kan G, Rolland Y, Bergman H, Morley JE, Kritchevsky SB, Vellas B. The I.A.N.A Task Force on frailty assessment of older people in clinical practice. J Nutr Health Aging. 2008 Jan;12(1):29-37.

10. Kaya G. and J. H. Saurat (2007). "Dermatoporosis: a chronic cutaneous insufficiency/fragility syndrome. Clinicopathological features, mechanisms, prevention and potential treatments." Dermatology 215(4): 284-94. 